\title{
Note on quantitative homogenization results for parabolic systems in $\mathbb{R}^{d}$
}

\author{
Yulia MeshKova(D
}

Abstract. In $L_{2}\left(\mathbb{R}^{d} ; \mathbb{C}^{n}\right)$, we consider a semigroup $e^{-t A_{\varepsilon}}, t \geqslant 0$, generated by a matrix elliptic secondorder differential operator $A_{\varepsilon} \geqslant 0$. Coefficients of $A_{\varepsilon}$ are periodic, depend on $\mathbf{x} / \varepsilon$, and oscillate rapidly as $\varepsilon \rightarrow 0$. Approximations for $e^{-t A_{\varepsilon}}$ were obtained by Suslina (Funktsional Analiz i ego Prilozhen 38(4):8690, 2004) and Suslina (Math Model Nat Phenom 5(4):390-447, 2010) via the spectral method and by Zhikov and Pastukhova (Russ J Math Phys 13(2):224-237, 2006) via the shift method. In the present note, we give another short proof based on the contour integral representation for the semigroup and approximations for the resolvent with two-parametric error estimates obtained by Suslina (2015).

\section{Introduction}

The subject of this note is quantitative estimates in periodic homogenization, i.e. approximations for the corresponding resolving operator in the uniform operator topology. There are several approaches to obtaining results of such type, see $[1,2,6,13]$.

In the introduction, let us consider the simplest elliptic operator $A_{\varepsilon}=$ $-\operatorname{div} g\left(\varepsilon^{-1} \mathbf{x}\right) \nabla, \varepsilon>0$, acting in $L_{2}\left(\mathbb{R}^{d}\right)$. Here, $g$ is a periodic positive definite matrix-valued function such that $g, g^{-1} \in L_{\infty}$. Let $u_{\varepsilon}$ be the solution of the equation $A_{\varepsilon} u_{\varepsilon}+u_{\varepsilon}=F$, where $F \in L_{2}\left(\mathbb{R}^{d}\right)$. The homogenization problem is to describe the behaviour of the solution $u_{\varepsilon}$ in the small period limit $\varepsilon \rightarrow 0$. The classical result is that $u_{\varepsilon} \rightarrow u_{0}$ in the $L_{2}$-norm, where the limit function $u_{0}$ is the solution of the equation of the same type $A^{0} u_{0}+u_{0}=F$, where $A^{0}=-\operatorname{div} g^{0} \nabla$ is the so-called effective operator with the constant matrix $g^{0}$.

By using the spectral method, M. Sh. Birman and T. A. Suslina [1] proved that $\left\|u_{\varepsilon}-u_{0}\right\|_{L_{2}} \leqslant C \varepsilon\|F\|_{L_{2}}$. This estimate can be rewritten as approximation for the resolvent $\left(A_{\varepsilon}+I\right)^{-1}$ in the uniform operator topology. Approximations for the semigroup $e^{-t A_{\varepsilon}}, t \geqslant 0$, were obtained in [7,8], and [12] via the spectral and shift methods,

Mathematics Subject Classification: Primary 35B27, Secondary 35K45

Keywords: Homogenization, Convergence rates, Parabolic systems, Trotter-Kato theorem.

Research was supported by «Native towns 》, a social investment programme of PJSC «Gazprom Neft》, by the postdoctoral fellowship of the Hausdorff Research Institute for Mathematics in Bonn, Germany, and, last but not least, by the SPbU travel money informally called "100 RUB from Eremeev". The work on the final version of the paper was sponsored by the European Research Council (ERC) under the European Union's Horizon 2020 research and innovation programme (Grant Agreement No. 818437). 
respectively:

$$
\left\|e^{-t A_{\varepsilon}}-e^{-t A^{0}}\right\|_{L_{2}\left(\mathbb{R}^{d}\right) \rightarrow L_{2}\left(\mathbb{R}^{d}\right)} \leqslant C \varepsilon\left(t+\varepsilon^{2}\right)^{-1 / 2} .
$$

Since the point zero is the lower edge of the spectra for $A_{\varepsilon}$ and $A^{0}$, estimate (1) can be treated as a stabilization result for $t \rightarrow \infty$. Later in [5], it was observed that quantitative results for parabolic problems can be derived from corresponding elliptic results with the help of identity $e^{-t A_{\varepsilon}}=-\frac{1}{2 \pi i} \int_{\gamma} e^{-\zeta t}\left(A_{\varepsilon}-\zeta I\right)^{-1} d \zeta$, where $\gamma \subset \mathbb{C}$ is a contour enclosing the spectrum of $A_{\varepsilon}$ in the positive direction. But in [5] only problems in a bounded domain $\mathcal{O} \subset \mathbb{R}^{d}$ were studied and operators under consideration were positive definite. In the case of the Dirichlet boundary condition, it was obtained that

$$
\left\|e^{-t A_{D, \varepsilon}}-e^{-t A_{D}^{0}}\right\|_{L_{2}(\mathcal{O}) \rightarrow L_{2}(\mathcal{O})} \leqslant C \varepsilon\left(t+\varepsilon^{2}\right)^{-1 / 2} e^{-c t} .
$$

The unique conceptual difference between (1) and (2) is the behaviour at $t \rightarrow \infty$. While (2) contains exponentially decaying factor $e^{-c t}$, we cannot speak about stabilization for $A_{D, \varepsilon}$. Indeed, the difference of the operator exponentials satisfies the rough estimate

$$
\left\|e^{-t A_{D, \varepsilon}}-e^{-t A_{D}^{0}}\right\| \leqslant 2 e^{-c_{*} t},
$$

where $c_{*}>0$ is a common lower bound for $A_{D, \varepsilon}$ and $A_{D}^{0}$. In (2), the constant $c$ is such that $0<c<c_{*}$ and the constant $C$ depends on our choice of $c$ and grows as $c \rightarrow c_{*}$. This is caused by the used Cauchy integral representation and the behaviour of the error estimate in approximation for the resolvent $\left(A_{D, \varepsilon}-\zeta I\right)^{-1}$ for small fixed $|\zeta|$. According to the results of [10], the error estimate for the resolvent $\left(A_{\varepsilon}-\zeta I\right)^{-1}$ has different behaviour with respect to $\zeta$ compared to the known one for $\left(A_{D, \varepsilon}-\zeta I\right)^{-1}$.

The goal of the present note is to show how parabolic results from $[7,9,12]$ can be derived from approximations for $\left(A_{\varepsilon}-\zeta I\right)^{-1}$ in $\left(L_{2} \rightarrow L_{2}\right)$ - and $\left(L_{2} \rightarrow H^{1}\right)$-norms from [10]. The difference between methods of the present paper and [5] consists of choosing the contour $\gamma$ depending on time $t$. This idea is inspired by the proof of [3, Lemma 1].

\section{Preliminaries. Known results}

Let $\Gamma \subset \mathbb{R}^{d}$ be a lattice, and let $\Omega$ be the cell of the lattice $\Gamma$. By $H_{\text {per }}^{1}(\Omega)$, we denote the subspace of matrix-valued functions from $H^{1}(\Omega)$ whose $\Gamma$-periodic extension belongs to $H_{\mathrm{loc}}^{1}\left(\mathbb{R}^{d}\right)$. For any $\Gamma$-periodic matrix-valued function $f$ we use the notation $f^{\varepsilon}(\mathbf{x}):=f\left(\varepsilon^{-1} \mathbf{x}\right), \varepsilon>0$. By $\left[f^{\varepsilon}\right]$, we denote the operator of multiplication by the matrix-valued function $f^{\varepsilon}(\mathbf{x})$.

In $L_{2}\left(\mathbb{R} ; \mathbb{C}^{n}\right)$, we consider a matrix elliptic second-order differential operator $A_{\varepsilon}$, $\varepsilon>0$, formally given by the expression $A_{\varepsilon}=b(\mathbf{D})^{*} g^{\varepsilon}(\mathbf{x}) b(\mathbf{D})$. Here $g$ is a $\Gamma$-periodic $(m \times m)$-matrix-valued function, $g(\mathbf{x})>0, g, g^{-1} \in L_{\infty}$, and $b(\mathbf{D})=\sum_{l=1}^{d} b_{l} D_{l}$ is a first-order differential operator whose coefficients $b_{l}, l=1, \ldots, d$, are constant 
$(m \times n)$-matrices. The entries of the matrices $g(\mathbf{x})$ and $b_{l}, l=1, \ldots, d$, are in general complex. Suppose that $m \geqslant n$ and that the symbol $b(\xi)=\sum_{l=1}^{d} b_{l} \xi_{l}$ satisfies the full rank condition: $\operatorname{rank} b(\xi)=n, 0 \neq \xi \in \mathbb{R}^{d}$. Or, equivalently, there exist constants $\alpha_{0}$ and $\alpha_{1}$ such that

$$
\alpha_{0} \mathbf{1}_{n} \leqslant b(\boldsymbol{\theta})^{*} b(\boldsymbol{\theta}) \leqslant \alpha_{1} \mathbf{1}_{n}, \quad \boldsymbol{\theta} \in \mathbb{S}^{d-1}, \quad 0<\alpha_{0} \leqslant \alpha_{1}<\infty .
$$

Under the above assumptions, the operator $A_{\varepsilon}$ is self-adjoint, non-negative and strongly elliptic. The precise definition of $A_{\varepsilon}$ is given via the corresponding quadratic form on $H^{1}\left(\mathbb{R}^{d} ; \mathbb{C}^{n}\right)$.

The simplest example of the operator under consideration is the acoustics operator $A_{\varepsilon}=-\operatorname{div} g^{\varepsilon}(\mathbf{x}) \nabla$. The operator of elasticity theory also can be written as $b(\mathbf{D})^{*} g^{\varepsilon}(\mathbf{x}) b(\mathbf{D})$, see details in [1, Chapter 5].

The coefficients of the operator $A_{\varepsilon}$ oscillate rapidly as $\varepsilon \rightarrow 0$. The limit behaviour of its resolvent or the semigroup $e^{-t A_{\varepsilon}}$ is given by the corresponding function of the so-called effective operator $A^{0}=b(\mathbf{D})^{*} g^{0} b(\mathbf{D})$ with the constant matrix $g^{0}$. The definition of $g^{0}$ is given in terms of the $\Gamma$-periodic $(n \times m)$-matrix-valued function $\Lambda$ :

$$
g^{0}=|\Omega|^{-1} \int_{\Omega} g(\mathbf{x})\left(b(\mathbf{D}) \Lambda(\mathbf{x})+\mathbf{1}_{m}\right) d \mathbf{x},
$$

where $\Lambda \in H_{p e r}^{1}(\Omega)$ is the weak solution of the cell problem

$$
b(\mathbf{D})^{*} g(\mathbf{x})\left(b(\mathbf{D}) \Lambda(\mathbf{x})+\mathbf{1}_{m}\right)=0, \quad \int_{\Omega} \Lambda(\mathbf{x}) d \mathbf{x}=0 .
$$

By $S_{\varepsilon}$, we denote the Steklov smoothing operator acting in $L_{2}\left(\mathbb{R}^{d} ; \mathbb{C}^{m}\right)$ by the rule

$$
\left(S_{\varepsilon} \mathbf{u}\right)(\mathbf{x})=|\Omega|^{-1} \int_{\Omega} \mathbf{u}(\mathbf{x}-\varepsilon \mathbf{z}) d \mathbf{z} .
$$

According to [11, Lemma 1.2], for any $\Gamma$-periodic function $f$ in $\mathbb{R}^{d}$ such that $f \in$ $L_{2}(\Omega)$, the operator $\left[f^{\varepsilon}\right] S_{\varepsilon}$ is continuous in $L_{2}\left(\mathbb{R}^{d}\right)$, and $\left\|\left[f^{\varepsilon}\right] S_{\varepsilon}\right\|_{L_{2}\left(\mathbb{R}^{d}\right) \rightarrow L_{2}\left(\mathbb{R}^{d}\right)} \leqslant$ $|\Omega|^{-1 / 2}\|f\|_{L_{2}(\Omega)}$. Using this fact and the inclusion $\Lambda \in H_{\mathrm{per}}^{1}(\Omega)$, one can show that the so-called corrector

$$
K(\varepsilon ; \zeta):=\left[\Lambda^{\varepsilon}\right] S_{\varepsilon} b(\mathbf{D})\left(A^{0}-\zeta I\right)^{-1}
$$

acts continuously from $L_{2}\left(\mathbb{R}^{d} ; \mathbb{C}^{n}\right)$ to $H^{1}\left(\mathbb{R}^{d} ; \mathbb{C}^{n}\right)$, and $\|K(\varepsilon ; \zeta)\|_{L_{2} \rightarrow H^{1}}=O\left(\varepsilon^{-1}\right)$ for fixed $\zeta \in \mathbb{C} \backslash \mathbb{R}_{+}$. The $\left(L_{2} \rightarrow H^{1}\right)$-continuity of the operator (9) below can be checked with the help of the same arguments.

The following result was obtained in [10, Theorems 2.2 and 2.4].

Theorem 1. ([10]) Let the above assumptions be satisfied. Let $\zeta \in \mathbb{C} \backslash \mathbb{R}_{+}, \phi=\arg \zeta$. Denote

$$
c(\phi)=\left\{\begin{array}{l}
|\sin \phi|^{-1}, \quad \phi \in(0, \pi / 2) \cup(3 \pi / 2,2 \pi), \\
1, \quad \phi \in[\pi / 2,3 \pi / 2] .
\end{array}\right.
$$


Then, for $\varepsilon>0$, we have

$$
\left\|\left(A_{\varepsilon}-\zeta I\right)^{-1}-\left(A^{0}-\zeta I\right)^{-1}\right\|_{L_{2}\left(\mathbb{R}^{d}\right) \rightarrow L_{2}\left(\mathbb{R}^{d}\right)} \leqslant C_{1} c(\phi)^{2}|\zeta|^{-1 / 2} \varepsilon .
$$

Let $K(\varepsilon ; \zeta)$ be the corrector (3). Then, for $\varepsilon>0$, we have

$$
\begin{aligned}
& \left\|\mathbf{D}\left(\left(A_{\varepsilon}-\zeta I\right)^{-1}-\left(A^{0}-\zeta I\right)^{-1}-\varepsilon K(\varepsilon ; \zeta)\right)\right\|_{L_{2}\left(\mathbb{R}^{d}\right) \rightarrow L_{2}\left(\mathbb{R}^{d}\right)} \leqslant C_{2} c(\phi)^{2} \varepsilon, \\
& \left\|\left(A_{\varepsilon}-\zeta I\right)^{-1}-\left(A^{0}-\zeta I\right)^{-1}-\varepsilon K(\varepsilon ; \zeta)\right\|_{L_{2}\left(\mathbb{R}^{d}\right) \rightarrow L_{2}\left(\mathbb{R}^{d}\right)} \leqslant C_{3} c(\phi)^{2}|\zeta|^{-1 / 2} \varepsilon .
\end{aligned}
$$

The constant $C_{1}$ depends only on $\alpha_{0}, \alpha_{1},\|g\|_{L_{\infty}},\left\|g^{-1}\right\|_{L_{\infty}}$, and parameters of the lattice $\Gamma$. The constants $C_{2}$ and $C_{3}$ depend on the same parameters and also on $m$ and d.

The aim of the present paper is to give another proof of the following theorem.

Theorem 2. ( $[7,9,12])$ Under the above assumptions, for $\varepsilon>0$ and $t \geqslant 0$ we have

$$
\left\|e^{-t A_{\varepsilon}}-e^{-t A^{0}}\right\|_{L_{2}\left(\mathbb{R}^{d}\right) \rightarrow L_{2}\left(\mathbb{R}^{d}\right)} \leqslant C_{4} \varepsilon\left(t+\varepsilon^{2}\right)^{-1 / 2} .
$$

Denote

$$
\mathcal{K}(\varepsilon ; t):=\left[\Lambda^{\varepsilon}\right] S_{\varepsilon} b(\mathbf{D}) e^{-t A^{0}} .
$$

Then for $\varepsilon>0$ and $t>0$ we have

$$
\begin{gathered}
\left\|\mathbf{D}\left(e^{-t A_{\varepsilon}}-e^{-t A^{0}}-\varepsilon \mathcal{K}(\varepsilon ; t)\right)\right\|_{L_{2}\left(\mathbb{R}^{d}\right) \rightarrow L_{2}\left(\mathbb{R}^{d}\right)} \leqslant C_{5} \varepsilon t^{-1}, \\
\left\|e^{-t A_{\varepsilon}}-e^{-t A^{0}}-\varepsilon \mathcal{K}(\varepsilon ; t)\right\|_{L_{2}\left(\mathbb{R}^{d}\right) \rightarrow L_{2}\left(\mathbb{R}^{d}\right)} \leqslant C_{6} \varepsilon t^{-1 / 2} .
\end{gathered}
$$

The constant $C_{4}$ depends only on $\alpha_{0}, \alpha_{1},\|g\|_{L_{\infty}},\left\|g^{-1}\right\|_{L_{\infty}}$, and parameters of the lattice $\Gamma$. The constants $C_{5}$ and $C_{6}$ depend on the same parameters and also on $m$ and $d$.

Remark 3. Estimate (8) was announced in [7, Theorem 1] and proved in [8, Theorem 7.1] and, for the acoustics operator, in [12, Theorem 1.1]. For the scalar elliptic operator $A_{\varepsilon}=-\operatorname{div} g^{\varepsilon}(\mathbf{x}) \nabla$, where $g(\mathbf{x})$ is a symmetric matrix with real entries, one has $\Lambda \in L_{\infty}$ and it is possible to replace the smoothing operator $S_{\varepsilon}$ in the corrector by the identity operator. In this case, estimate (10) was obtained in [12, Theorem 1.3]. For the matrix elliptic operator, $\left(L_{2} \rightarrow H^{1}\right)$-approximation for $e^{-t A_{\varepsilon}}$ was proved in [9, Theorem 11.1] (but with another smoothing operator in the corrector).

\section{New proof}

Using the Riesz-Dunford functional calculus, we represent the operator exponential $e^{-t A_{\varepsilon}}$ as an integral:

$$
e^{-t A_{\varepsilon}}=-\frac{1}{2 \pi i} \int_{\gamma} e^{-\zeta t}\left(A_{\varepsilon}-\zeta I\right)^{-1} d \zeta .
$$


Here, $\gamma$ is a suitable contour in the complex plain enclosing the spectrum $\sigma\left(A_{\varepsilon}\right) \subset$ $[0, \infty)$ in the positive direction. One can choose $\gamma=\widehat{\gamma} \cup \tilde{\gamma}$ with

$$
\begin{aligned}
& \widehat{\gamma}=\left\{\zeta \in \mathbb{C}: \zeta=e^{i \phi}, \pi / 4 \leqslant \phi \leqslant 7 \pi / 4\right\}, \\
& \tilde{\gamma}=\left\{\zeta \in \mathbb{C}: \zeta=r e^{i \pi / 4}, r \geqslant 1\right\} \cup\left\{\zeta \in \mathbb{C}: \zeta=r e^{i 7 \pi / 4}, r \geqslant 1\right\} .
\end{aligned}
$$

But we take the contour depending on $t>0$, shrinking this contour $\gamma$ in $t$ times: $\gamma_{t}=t^{-1} \gamma=\left\{\zeta \in \mathbb{C}: \zeta=t^{-1} \eta, \eta \in \gamma\right\}$. Applying these arguments to the operators $e^{-t A_{\varepsilon}}$ and $e^{-t A^{0}}$ and changing variable, we get

$$
\begin{aligned}
e^{-t A_{\varepsilon}}-e^{-t A^{0}} & =-\frac{1}{2 \pi i} \int_{\gamma_{t}} e^{-\zeta t}\left(\left(A_{\varepsilon}-\zeta I\right)^{-1}-\left(A^{0}-\zeta I\right)^{-1}\right) d \zeta \\
& =-\frac{1}{2 \pi i t} \int_{\gamma} e^{-\eta}\left(\left(A_{\varepsilon}-t^{-1} \eta I\right)^{-1}-\left(A^{0}-t^{-1} \eta I\right)^{-1}\right) d \eta
\end{aligned}
$$

Recall notation (4). Using (5) and taking into account that $c\left(\phi_{t}\right) \leqslant 2^{1 / 2}$, where $\eta \in \gamma$ and $\phi_{t}:=\arg \left(t^{-1} \eta\right)$, for $t>0$ we have

$$
\left\|e^{-t A_{\varepsilon}}-e^{-t A^{0}}\right\|_{L_{2}\left(\mathbb{R}^{d}\right) \rightarrow L_{2}\left(\mathbb{R}^{d}\right)} \leqslant \frac{C_{1} \varepsilon}{\pi t} \int_{\gamma}\left|e^{-\eta}\right|\left|t^{-1} \eta\right|^{-1 / 2}|d \eta| \leqslant \frac{C_{1} \varepsilon}{\pi t^{1 / 2}} \int_{\gamma}\left|e^{-\eta}\right||d \eta| .
$$

The integral here is understood as a contour integral of the first kind. Let us estimate it:

$$
\frac{1}{\pi} \int_{\gamma}\left|e^{-\eta}\right||d \eta| \leqslant \frac{1}{\pi} \int_{\pi / 4}^{7 \pi / 4} e^{-\cos \phi} d \phi+\frac{2}{\pi} \int_{1}^{\infty} e^{-r / \sqrt{2}} d r \leqslant 3 e / 2+2^{3 / 2} \pi^{-1} e^{-1 / \sqrt{2}}=: \mathfrak{c} .
$$

Thus, $\left\|e^{-t A_{\varepsilon}}-e^{-t A^{0}}\right\|_{L_{2}\left(\mathbb{R}^{d}\right) \rightarrow L_{2}\left(\mathbb{R}^{d}\right)} \leqslant \mathfrak{c} C_{1} \varepsilon t^{-1 / 2}$. Obviously, for $t \geqslant 0$ the left-hand side of (13) does not exceed 2. Since $\min \left\{2 ; \mathfrak{c} C_{1} \varepsilon t^{-1 / 2}\right\} \leqslant C_{4} \varepsilon\left(t+\varepsilon^{2}\right)^{-1 / 2}$, where $C_{4}=2^{1 / 2} \max \left\{2 ; \mathfrak{c} C_{1}\right\}$, we arrive at estimate (8).

To prove $\left(L_{2} \rightarrow H^{1}\right)$-approximation in the same manner, we need an identity for the correctors (3) and (9). So, we act by the operator $\left[\Lambda^{\varepsilon}\right] S_{\varepsilon} b(\mathbf{D})$ from the left to the both sides of the contour integral representation for the exponential $e^{-t A^{0}}$. Since the operator $\left[\Lambda^{\varepsilon}\right] S_{\varepsilon} b(\mathbf{D})$ is closed, we can move it across the integral sign. Thus,

$$
\mathcal{K}(\varepsilon ; t)=-\frac{1}{2 \pi i} \int_{\gamma_{t}} e^{-\zeta t} K(\varepsilon ; \zeta) d \zeta
$$

Similarly to the proof of estimate (8), relations (6), (12), (14), and (15) imply estimate (10) with the constant $C_{5}:=\mathfrak{c} C_{2}$. Estimate (11) follows from (7) on the same way, $C_{6}:=\mathfrak{c}_{3}$.

\section{Discussion}

Since we derive the parabolic estimates from the elliptic ones, the achievement of the present paper can be interpreted as a quantitative Trotter-Kato like result in 
homogenization context. For derivation of hyperbolic results from elliptic ones, see preprint [4].

The author believes that the used technique may be useful for positive definite operators after refinement of the known resolvent estimates near the lower edge of the spectrum.

\section{Acknowledgements}

Open access funding provided by University of Helsinki including Helsinki University Central Hospital. The author is happy to thank the Hausdorff Research Institute for Mathematics in Bonn, Germany, for financial support, hospitality, and excellent working conditions during the Junior Trimester Program "Randomness, PDEs and Nonlinear Fluctuations". The author has to thank prof. T. A. Suslina for her attention to the work.

Open Access. This article is licensed under a Creative Commons Attribution 4.0 International License, which permits use, sharing, adaptation, distribution and reproduction in any medium or format, as long as you give appropriate credit to the original author(s) and the source, provide a link to the Creative Commons licence, and indicate if changes were made. The images or other third party material in this article are included in the article's Creative Commons licence, unless indicated otherwise in a credit line to the material. If material is not included in the article's Creative Commons licence and your intended use is not permitted by statutory regulation or exceeds the permitted use, you will need to obtain permission directly from the copyright holder. To view a copy of this licence, visit http://creativecommons.org/licenses/ by/4.0/.

Publisher's Note Springer Nature remains neutral with regard to jurisdictional claims in published maps and institutional affiliations.

\section{REFERENCES}

[1] M. Sh. Birman and T. A. Suslina, Second order periodic differential operators. Threshold properties and homogenization, Algebra i Analiz 15 (2003), no. 5, 1-108; English transl., St. Petersburg Math. J. 15 (2004), no. 5, 639-714.

[2] D. Cioranescu, A. Damlamian, and G. Griso, The periodic unfolding method. Theory and applications to partial differential problems, Springer, Series in Contemporary Mathematics, vol. 3, 2018.

[3] T. Ichinose, H. Neidhardt, and V. A. Zagrebnov, Trotter-Kato product formula and fractional powers of self-adjoint generators, J. Funct. Anal. 207 (2004), 33-57.

[4] Yu. Meshkova, Variations on the theme of the Trotter-Kato theorem for homogenization of periodic hyperbolic systems, arXiv:1904.02781 (2019).

[5] Yu. M. Meshkova and T. A. Suslina, Homogenization of initial boundary value problems for parabolic systems with periodic coefficients, Applicable Analysis 95:8 (2016), 1736-1775.

[6] Z. Shen, Periodic homogenization of elliptic systems, Advances in Partial Differential Equations, Oper. Theory Adv. Appl., vol. 296, Birkhäuser/Springer, 2018.

[7] T. A. Suslina, On homogenization of periodic parabolic systems, Funktsional. Funct Analiz i ego Prilozhen. 38 (2004), no. 4, 86-90; English transl., Funct. Anal. Appl. 38 (2004), no. 4, 309-312.

[8] T. A. Suslina, Homogenization of a periodic parabolic Cauchy problem, Nonlinear equations and spectral theory, 201-233, Amer. Math. Soc. Transl. (2), vol. 220, Amer. Math. Soc., Providence, RI, 2007. 
[9] T. A. Suslina, Homogenization of a periodic parabolic Cauchy problem in the Sobolev space $H^{1}\left(\mathbb{R}^{d}\right)$, Math. Model. Nat. Phenom. 5 (2010), no. 4, 390-447.

[10] T. A. Suslina, Homogenization of elliptic operators with periodic coefficients in dependence of the spectral parameter, Algebra i Analiz 27 (2015), no. 4, 87-166; English transl., St. Petersburg Math. J. 27 (2016), no. 4, 651-708.

[11] V. V. Zhikov and S. E. Pastukhova, On operator estimates for some problems in homogenization theory, Russ. J. Math. Phys. 12 (2005), no. 4, 515-524.

[12] V. V. Zhikov and S. E. Pastukhova, Estimates of homogenization for a parabolic equation with periodic coefficients, Russ. J. Math. Phys. 13 (2006), no. 2, 224-237.

[13] V. V. Zhikov and S. E. Pastukhova, Operator estimates in homogenization theory, Uspekhi Matem. Nauk 71 (429) (2016), no. 3, 27-122; English transl., Russian Math. Surveys 71 (2016), no. 3, 417-511.

\author{
Yulia Meshkova \\ Chebyshev Laboratory \\ St. Petersburg State University \\ 14th Line V.O., 29 \\ Saint Petersburg \\ Russia 199178 \\ and
}

Department of Mathematics and Statistics University of Helsinki

Pietari Kalmin katu 5

P.O. Box 68

o0014 Helsinki

Finland

E-mail:y.meshkova@spbu.ru; iuliia.meshkova@helsinki.fi 